\title{
Sleep-Wake Patterns during the Acute Phase after First-Ever Stroke
}

\author{
Linda N. Bakken, ${ }^{1,2}$ Kathryn A. Lee, ${ }^{3}$ Hesook Suzie Kim, ${ }^{1}$ \\ Arnstein Finset, ${ }^{2}$ and Anners Lerdal ${ }^{4,5}$ \\ ${ }^{1}$ Department of Health Sciences, Buskerud University College, P.O. Box 7053, 3007 Drammen, Norway \\ ${ }^{2}$ Department of Behavioral Medicine, Faculty of Medicine, University of Oslo, 0316 Oslo, Norway \\ ${ }^{3}$ Department of Family Health Care Nursing, University of California at San Francisco, San Francisco, CA 94143-0606, USA \\ ${ }^{4}$ Centre for Clinical Nursing Research, Lovisenberg Deaconal University College, 0456 Oslo, Norway \\ ${ }^{5}$ Research Centre and Division of Medicine, Department of Gastroenterology, Oslo University Hospital, 0424 Oslo, Norway
}

Correspondence should be addressed to Linda N. Bakken, linda.bakken@hibu.no

Received 29 September 2010; Revised 21 February 2011; Accepted 13 April 2011

Academic Editor: Alison Baird

Copyright ( $) 2011$ Linda N. Bakken et al. This is an open access article distributed under the Creative Commons Attribution License, which permits unrestricted use, distribution, and reproduction in any medium, provided the original work is properly cited.

\begin{abstract}
This study describes the pattern of day and night sleep and explores relationships between these patterns and sociodemographic and clinical factors as well as sleep environmental context and the patient's subjective sleep quality. Data from 110 patients with first-ever stroke was collected by structured interview surveys, medical record, and objective estimated sleep data from wrist actigraphy. The variability in estimated sleep is large. Half the patients slept either $<6$ hours or $>8$ hours per night, and $78 \%$ had more than nine awakenings per night. Men slept less than women, and patients sleeping at home had fewer awakenings than those who slept in hospital. It was estimated sleep during daytime in all, except 4, patients. Longer stay in hospital was related to more daytime sleep, and the subjective sleep quality correlated with estimated sleep time, wake time, and wake percentage.
\end{abstract}

\section{Introduction}

Clinicians frequently observe stroke patients with disturbed sleep in hospital, and there is a notable lack of studies describing their sleep-wake patterns [1]. Polysomnography (PSG) studies of the sleep of stroke patients during night time have reported both insomnia (difficulty falling asleep and staying asleep at night) and hypersomnia (inability to stay awake and excessive daytime sleepiness) [1-5].

Poor sleep efficiency and wakefulness after sleep onset are associated with reduced cognitive function in the acute phase after stroke [6] and nonrestorative sleep is described as restless, light, or poor-quality sleep, even when its duration appears normal. Poor sleep is also associated with daytime impairment [7]. After a stroke, restorative sleep is essential to gain physical and psychological energy, but it must be taken into consideration that age and numbers of medical condition influence patients' sleep quality $[8,9]$.

The aims of this study were to describe the sleep of patients in the acute phase after their first-ever stroke and to explore the relationships between sleep and sociodemographic variables, clinical variables, the patient's environmental context for sleep and self-reported sleep quality. There were two specific research questions.

(1) What are the night and day sleep-wake patterns during the acute phase (first 15 days) after a first-ever stroke?

(2) How are the night and day sleep-wake pattern related to sociodemographic variables (age, sex, education, cohabitation, and employment status), clinical variables (physical functioning, use of sedatives, and time since admission), environmental sleep context (sleep location), and self-reported sleep quality?

\section{Materials and Methods}

The study was a descriptive cross-sectional design. A sample of patients was recruited sequentially from two hospital wards in Norway. The design included both subjective and 
BHD

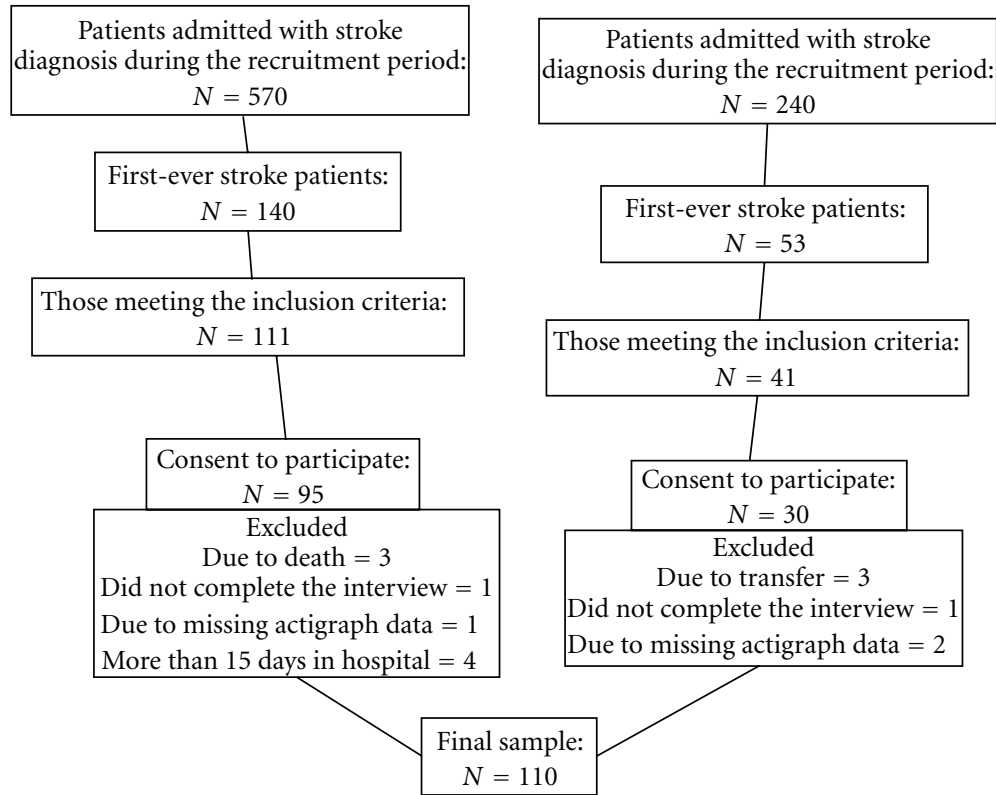

FIGURE 1: Flow chart for the sample recruitment.

objective measures within the first two weeks after diagnosis of a first-ever stroke.

2.1. Sampling. Patients admitted to one of two hospitals in Oslo and Buskerud County in Norway with a diagnosis of first-ever stroke from March 2007 to September 2008 were recruited for participation. The inclusion criteria were firstever clinical presentation of stroke according to International Classification of Diseases (ICD)-10 (I 60, 61, 62, 63, and 64) [10], satisfactory cognitive function and age over 18 years. To be included, a patient had to be fully conscious or able to awaken to full consciousness, and be oriented to time, place, and person. Patients unable to participate in a meaningful conversation or to point to the response alternatives on a questionnaire were excluded. Of the 193 patients with a diagnosis of first-ever stroke admitted to these two hospitals, 41 patients were excluded, because they had reduced cognitive function $(n=14)$, found communication difficult $(n=26)$, or did not understand Norwegian $(n=$ 1). Of the 152 patients who met inclusion criteria, 125 consented to participate; three patients died, and three were transferred to other regions. In the final sample of 119 patients in the study, all except one were ethnically White (see Figure 1).

Nine patients were excluded from analysis due to not completing the interview $(n=2)$, missing actigraph data $(n=3)$, and interview data collected after the acute phase $(n=4)$. Therefore, the sample for analysis included 110 patients.

\subsection{Measurements}

2.2.1. Sociodemographic Variables. Data were collected on age (years), sex, level of formal education (dichotomized as
$<12$ and $\geq 12$ years), coresidence status (paired relationship or not), and employment status.

2.2.2. Stroke Type and Location. Computed tomography (CT) scans were taken of all the patients at admission to the hospital. If an additional CT scan were taken, the latest description was used for categorizing. Based on the radiologists' descriptions, stroke type was grouped into the following four groups: (a) ischemic infarct, (b) hemorrhage (c) chronic cerebral ischemia, and (d) negative findings. Stroke location was grouped into left, right, and bilateral. Some of the CT scans showed signs of lesions from previous strokes that were undiagnosed. These lesions were included in the classification of stroke (see Table 1).

2.2.3. Clinical Variables. Physical functioning (PF) was measured on a 10-item subscale of the SF-36-Acute-Form for HRQoL [11] ranging from 0-100, where the higher scores represent higher quality of life (QoL). Cronbach's $\alpha$ was 0.94 for the PF items. Sedatives and sleeping medications were assessed as part of the same drug use category, as both influence the patient's sleep. Time since admission referred to the number of days that the patient had been in hospital when the first part of the interview was performed.

2.2.4. Sleep Environment Location. During hospitalization, some patients were given permission to sleep at home, especially in the weekends. We recorded their place of sleep and grouped them as those who slept in the hospital, those who slept at home, or those who only slept in the hospital for one or two nights.

2.2.5. Subjective Sleep Quality. Subjective sleep quality was assessed by the pittsburgh sleep quality index (PSQI) [12]. 
A sum score of PSQI is calculated, and ranges from 021 , where higher score indicates worse sleep quality. The internal consistency of the items and the test-retest reliability in the original version (in medical patients) [13], and in the Norwegian version have been reported to be satisfactory [14].

2.2.6. Night and Day Sleep-Wake Time and Pattern. Objective sleep was estimated with the Motion logger Actigraph (AA32 Ambulatory Monitoring Inc., Ardsley, NY, USA), a watchlike device that measures activity from the wrist. It is a valid and reliable noninvasive instrument for assessing sleep-wake behavior over 24 hours for several days in a natural sleeping environment [15]. It was worn on the patient's wrist on the unaffected arm. The actigraph was set to one-minute epochs using zero-crossing mode. It records activity in a continuous format. The activity counts were analyzed in Hertz $(\mathrm{Hz})$ with the Cole-Kripke algorithm from the ActionW software program, version 2.4 (Ambulatory Monitoring Inc.). The algorithm used a 2-minute rule to define a wake period. This wrist actigraphy method of estimating sleep has been shown to have a correspondence of $91-93 \%$ with PSG in adult populations [15]. The actigraph was fixed with a plastic bracelet so that it could not be removed without cutting. The analysis of sleep-wake pattern was based on continuous wrist actigraphy data for three nights and two days. Since the event maker could not be relied upon in this sample to indicate lights out at night or final wake time, sleep variables included any day sleep time between 09:00 and 20:59 (NAP minutes), sleep at night (TST minutes), number of wake episodes after sleep onset (WAKES), and wake after sleep onset (WASO) as minutes (WASO-M) as well as the percentage of total sleep time (WASO-P). Night sleep measures of TST, WAKES, and WASO between 21:00 and $08: 59$ were analyzed and the mean of the three nights were used for analysis. Day-time sleep was analyzed as the mean of the two 12-hr day intervals. The actigraph was programmed to start at 18:00, and data collected before 21:00 were discarded to allow the patients to adapt to wearing the monitor. Monitoring was performed on all days of the week, and ended at 09:00 on the third day.

2.3. Procedure. Data on age, sex, the use of medications, and time since admission were collected from the patient's medical record. Data on physical functioning were collected through interviews using standardized questionnaires. To ease the burden on the patients, the interviews were divided into two sessions of approximately 30 minutes each, the first and last day. The actigraph was placed on the patient's unaffected wrist in the first session and taken off in the second session.

2.4. Statistical Analysis. SPSS for Windows version 17.0 software (SPSS Inc., Ill, USA) was used for the analysis. Continuous sleep variables that were not initially normally distributed were log-transformed (and in cases of containing zero added one before log-transformation) so that parametric statistics could be used. Independent $t$-test $(t)$,
TABLE 1: Characteristics of the sample $(N=110)$.

\begin{tabular}{|c|c|c|c|c|}
\hline & Mean & $(\mathrm{SD})$ & Median & Range \\
\hline \multicolumn{5}{|l|}{ Sociodemographic variables } \\
\hline Age in years & 68.3 & $(13.2)$ & 70 & $29-91$ \\
\hline Males & 66.5 & $(13.0)$ & 68 & $29-86$ \\
\hline Females & 71.1 & $(13.1)$ & 74 & $37-91$ \\
\hline Education & $N$ & $(\%)$ & & \\
\hline$<12$ years (high) & 80 & (73) & & \\
\hline$\geq 12$ years $($ low $)$ & 30 & $(27)$ & & \\
\hline Living in paired relationship & 56 & $(50)$ & & \\
\hline Working status: paid job & 33 & $(30)$ & & \\
\hline \multicolumn{5}{|l|}{ Stroke type } \\
\hline Ischemic infarct & 78 & $(70.9)$ & & \\
\hline Hemorrhage & 6 & $(5.5)$ & & \\
\hline Chronic cerebral ischemia & 10 & $(14.5)$ & & \\
\hline Negative findings & 16 & $(9.1)$ & & \\
\hline \multicolumn{5}{|l|}{ Clinical variables } \\
\hline $\begin{array}{l}\text { Physical functioning } \\
\text { (from SF-36), (0-100) }\end{array}$ & 60.0 & $(33.7)$ & 70 & $0-100$ \\
\hline Time since admission & 4.6 & $(3.2)$ & 4 & $1-15$ \\
\hline Context & $N$ & $(\%)$ & & \\
\hline Hospital all nights & 54 & (49) & & \\
\hline Home all nights & 32 & (29) & & \\
\hline Part-time hospital & 24 & $(22)$ & & \\
\hline \multicolumn{5}{|l|}{ Subjective sleep quality } \\
\hline PSQI (0-21) & 6.9 & $(3.6)$ & 6 & $0-16$ \\
\hline
\end{tabular}

Chi-square test $(\chi)$, Pearson's correlation coefficient $(r)$, one-way ANOVA analyses with Tukey post hoc test and hierarchical multiple regression analysis $(\beta)$ were used to assess the relationships between the variables. All tests were two-tailed, and the level of significance was set at $P<$ .05. Cronbach's $\alpha$ was used to test the internal consistency of the questionnaires. Effect sizes were calculated on the dimensional differences between the groups according to Cohen's coefficient $(d)$ and $d$ values $\geq 0.40$ were considered clinically significant [16].

2.5. Ethics. The study was approved by the Regional Committee for Medical Research Ethics in Eastern Norway, the "Personvern ombud" at one of the hospitals and the Norwegian Data Inspectorate. Patients gave their written informed consent before participation.

\section{Results}

3.1. Demographic Characteristics. The mean age of the patients in the sample $(M=68.3 \pm 13.2)$ was not significantly different from those who did not want to participate and those who were excluded due to missing responses $(M=$ $70.6 \pm 11.3, P=.36)$. The proportion of men was higher in the sample $(n=72,60.5 \%)$ than among those who were not included $(n=8,32.0 \%, \chi=7.0$, df $=1, P=.009)$. 
TABLE 2: Night and day characteristics $(N=110)$.

\begin{tabular}{lcccc}
\hline & Mean & (SD) & Median & Range \\
\hline Night Sleep Variables & & & & \\
Total sleep (minutes) & 426 & $(107.4)$ & 441 & $26-609$ \\
Number of WAKES & 13.5 & $(6.1)$ & 13.5 & $3-40$ \\
Wake time (minutes) $^{*}$ & 167 & $(105.1)$ & 139 & $15-590$ \\
Wake \% during night $^{*}$ & 27.3 & $(16.8)$ & 22.9 & $2.7-$ \\
& & &
\end{tabular}

Daytime Sleep Variable

$\begin{array}{lllll}\text { Nap (minutes) })^{\dagger} & 85 & (89.2) & 61 & 0-390\end{array}$

*After first sleep onset, until last sleep onset; night time (21:00-08:59); average of three consecutive nights.

${ }^{\dagger}$ During daytime (09:00-20:59); average of two consecutive days.

Data on age and sex were missing for 8 patients among the nonparticipants. The sociodemographic variables, stroke type, other clinical variables, sleep location and subjective sleep quality are detailed in Table 1.

3.2. Stroke Type and Location. The data showed a high representation of ischemic infarcts, with 30 in the right hemisphere, 28 in the left, and 20 bilateral. Of the 6 hemorrhages, 3 were in the right and 3 in the left hemisphere.

3.3. Admission. The first part of the interview was held within the four days after admission for 67 (60.9\%) of the participants, between days 5 and 10 for 35 participants $(31.8 \%)$, and between days 11 and 15 for 8 participants $(7.3 \%)$.

3.4. Estimated Sleep-Wake Patterns and Sleep Variables. Details on characteristics for sleep night and day see Table 2. A categorization on sleep and wakes is listed in Table 3.

The majority $(54.5 \%)$ were estimated with less than 450 minutes of sleep during night time. Men had estimated sleep at an average of $411( \pm 117.4)$ minutes, while women had an average of $450( \pm 85.5)$ minutes estimated sleep per night. The range of WAKES was normally distributed from 3 to 40 times, with a median and a mean of 13.5 awakenings, but $25 \%$ had more than 16 awakenings per night. One-fourth of the patients $(25 \%)$ were awake for less than 96 minutes or less than $16 \%$ of the night, and $25 \%$ were awake for more than 205 minutes or $33 \%$ of the night. In only four patients (4 men) the actigraphs did not estimate any sleep during the daytime on either of the two days of monitoring.

Twenty-nine patients (26\%) took sedatives or sleep medication at night time while they wore the actigraphs.

3.5. Bivariate Associations. One-way ANOVA analyses showed no significant differences when the mean scores for the four CT groups were compared in relation to TST $(F=1.46, P=.23)$, WAKES $(F=0.61, P=.61)$, WASO-M $(F=1.63, P=.19)$, WASO-P $(F=0.54, P=.66)$, and NAP $(F=0.13, P=.94)$. Post hoc tests for all these analyses showed no differences among the different subgroups (all $P>.18$ ). The ANOVA analysis for different infarct
TABLE 3: Sleep, wakes, and naps in categories $(N=110)$.

\begin{tabular}{lcccc}
\hline & $N(\%)$ & Mean & $(\mathrm{SD})$ & Range \\
\hline Sleep (hours) & & (Minutes) & & $($ Minutes $)$ \\
Less than 6 & $23(21)$ & 266 & 93.5 & $25.7-355.7$ \\
6-8 hours & $55(50)$ & 431 & 32.8 & $\begin{array}{c}361.0- \\
478.3\end{array}$ \\
& & & & $480.3-$ \\
More than 8 & $32(29)$ & 534 & 38.8 & 609.0 \\
\hline Wakes (numbers) & & $($ Numbers $)$ & & $($ Numbers $)$ \\
Less than 9 & $25(23)$ & 6 & $(1.9)$ & $3-8.7$ \\
9-16 & $59(54)$ & 13 & $(2.3)$ & $9-16.7$ \\
17 and more & $26(24)$ & 21 & $(5.4)$ & $17-40.3$ \\
\hline Nap (minutes) & & $($ Minutes $)$ & & $($ Minutes $)$ \\
0 & $4(3.6)$ & 0 & $(0.0)$ & 0 \\
1-30 & $33(30.0)$ & 15 & $(8.4)$ & $4-29.5$ \\
31-60 & $18(16.4)$ & 47 & $(7.3)$ & $33-60$ \\
61-90 & $16(14.5)$ & 78 & $(9.1)$ & $61.5-90$ \\
91-120 & $17(15.5)$ & 103 & $(9.1)$ & $91-118.5$ \\
$121-150$ & $5(4.5)$ & 134 & $(8.1)$ & $122-143$ \\
$151-$ more & $17(15.5)$ & 255 & $(85.6)$ & $154-389.5$ \\
\hline
\end{tabular}

locations indicated no differences among the groups for TST $(F=1.24, P=.88)$, WAKES $(F=1.13, P=.29)$, WASO-M $(F=0.28, P=.76)$, WASO-P $(F=0.18, P=.84)$, and NAP $(F=0.30, P=.74)$. The post hoc test for all these analyses showed no differences among the different subgroups (all $P>.33$ ). When we compared mean scores between the right and the left location of the hemorrhage locations no differences were found with regards to TST $(t=0.93, P=.41)$, WAKES $(t=1.93, P=.13)$, WASO-M $(t=-2.62, P=.06)$, WASO-P $(t=-0.61, P=.58)$, and $\operatorname{NAP}(t=-0.84, P=.45)$.

The correlation analysis (Table 4 ) showed that a longer stay in hospital was related to more daytime sleep $(r=0.19$, $P=.04$ ). Poor self-reported sleep quality (higher PSQI score) was related negatively with TST $(r=--0.19, P=$ $.042)$ and positively with WASO-M $(r=0.25, P=.02)$ and WASO-P ( $r=0.25, P=.02)$ by actigraphy.

Patients who slept all three nights at home had fewer mean WAKES $(M=11.1 \pm 4.69)$ compared with those who slept all three nights at the hospital $(M=14.8 \pm 6.77$, $t=2.74, P=.007, d=0.64)$. There was also a tendency for less WAKES among those sleeping all three nights at home compared to those who slept one or two nights at the hospital during data collection $(M=13.7 \pm 5.18, t=-1.96, P=.055$, $d=0.53)$. Twenty three men $(M=64.7 \pm 11.17$ years $)$ and 9 women $(M=69.3 \pm 12.19$ years $)$ slept at home all three nights.

3.6. Multivariate Analysis. The multivariate analysis showed that men had less estimated sleep than women during night time $(P=.047)$ (Table 5). Patients sleeping all three nights at home had fewer WAKES $(P=.007)$ than patients sleeping at the hospital for one, two or all three nights, even after controlling for sociodemographic and clinical variables. 
TABLE 4: Bivariate correlations (Pearson's $r$ ) between socsiodemographic and clinical variables $(N=110)$.

\begin{tabular}{lccccc}
\hline $\begin{array}{l}\text { Sociodemographic and } \\
\text { clinical variables }\end{array}$ & $\begin{array}{c}\text { Total sleep in } \\
\text { minutes }\end{array}$ & $\begin{array}{c}\text { Number of } \\
\text { awakenings }\end{array}$ & $\begin{array}{c}\text { Wake time in } \\
\text { minutes }\end{array}$ & $\begin{array}{c}\text { Wake of } \\
\text { TST }\end{array}$ & $\begin{array}{c}\text { Daytime } \\
\text { sleep }\end{array}$ \\
\hline & $r$ & $r$ & $r$ & $r$ & 0.04 \\
$\begin{array}{l}\text { Age } \\
\begin{array}{l}\text { Physical functioning } \\
\text { (SF-36-PF) }\end{array}\end{array}$ & 0.15 & 0.17 & 0.04 & -0.13 & -0.14 \\
$\begin{array}{l}\text { Time since admission } \\
\text { (days) }\end{array}$ & 0.01 & -0.15 & -0.13 & -0.02 & $0.19^{*}$ \\
Sleep quality (PSQI global) & 0.02 & 0.03 & 0.04 & $0.25^{\dagger}$ & 0.04 \\
\hline
\end{tabular}

${ }^{*} P<.05,{ }^{\dagger} P<.01$.

TABLE 5: Multivariate analysis (linear regression) of the possible predictors (sociodemographic, clinical variables and context) of sleep-wake patterns with total sleep time, number of wakes, wake in percent, and minutes sleep at daytime as dependent variables (standardized beta, $N=106)$.

\begin{tabular}{|c|c|c|c|c|c|c|c|c|}
\hline & \multicolumn{2}{|c|}{ Total sleep time (TST) } & \multicolumn{2}{|c|}{ Wakes } & \multicolumn{2}{|c|}{ Wake $\%$ of TST } & \multicolumn{2}{|c|}{ Daytime sleep } \\
\hline & $\beta$ & $P$ & $\beta$ & $P$ & $\beta$ & $P$ & $\beta$ & $P$ \\
\hline \multicolumn{9}{|l|}{ Step 1. Sociodemographic } \\
\hline Age & 0.19 & .18 & 0.15 & .30 & -0.03 & .85 & -0.02 & .99 \\
\hline Sex (men as ref.) & 0.21 & .047 & -0.13 & .21 & -0.11 & .31 & 0.11 & .31 \\
\hline Education (low, high) & 0.05 & .60 & 0.03 & .77 & -0.02 & .88 & 0.03 & .75 \\
\hline Paired relationship (paired as ref.) & -0.09 & .41 & -0.12 & .24 & -0.06 & .56 & 0.08 & .44 \\
\hline Work (paid work as ref.) & -0.05 & .74 & 0.04 & .78 & 0.01 & .96 & 0.07 & .60 \\
\hline Explained variance $\left(R^{2}\right)$ & $7.2 \%$ & & $5.7 \%$ & & $0.7 \%$ & & $3.6 \%$ & \\
\hline \multicolumn{9}{|l|}{ Step 2. Clinical } \\
\hline Physical functioning (SF-36-PF) & 0.02 & .89 & -0.06 & .56 & -0.08 & .47 & -0.08 & .49 \\
\hline Sedatives & 0.00 & .99 & -0.01 & .95 & 0.14 & .19 & -0.08 & .47 \\
\hline Time since admission (days) & 0.05 & .63 & -0.05 & .64 & -0.11 & .30 & 0.19 & .08 \\
\hline Explained variance $\left(R^{2}\right)$ & $7.5 \%$ & & $7.3 \%$ & & $4.3 \%$ & & $8.5 \%$ & \\
\hline$R^{2}$ change & $\mathbf{0 . 3} \%$ & & $1.6 \%$ & & $3.7 \%$ & & $4.9 \%$ & \\
\hline \multicolumn{9}{|l|}{ Step 3. Context } \\
\hline $\begin{array}{l}\text { Slept at home all nights } \\
\text { (hospital as ref.) }\end{array}$ & 0.10 & .38 & -0.32 & .007 & -0.21 & .09 & -0.02 & .84 \\
\hline $\begin{array}{l}\text { Slept both at hospital and home } \\
\text { (hospital as ref.) }\end{array}$ & 0.13 & .25 & -0.10 & .39 & -0.06 & .58 & -0.07 & .56 \\
\hline Explained variance $\left(R^{2}\right)$ & $8.9 \%$ & & $14.3 \%$ & & $7.2 \%$ & & $8.9 \%$ & \\
\hline$R^{2}$ change & $1.4 \%$ & & $7.0 \%$ & & $2.9 \%$ & & $0.3 \%$ & \\
\hline
\end{tabular}

WASO-P represents a standardized measurement of the time awake relative to the time spent in bed and was, therefore, chosen for the multivariate analysis. After controlling for demographic variables, the clinical variables only accounted for an additional $0.3 \%$ of the variance in TST, $1.6 \%$ of the variation in WAKES, 3.7\% of the variance in WASO-P, and $4.9 \%$ of the variation in NAP. When the environmental sleep context was then added into the model, it accounted for an additional $1.4 \%$ of the variance in TST, $7.0 \%$ of the variance in WAKES, $2.9 \%$ of the variance in WASO-P, and only $0.3 \%$ of the variance in NAP.

\section{Discussion}

This is the first study to our knowledge which describes the sleep-wake patterns in stroke patients during the acute phase.
Although the estimated mean TST (426 minutes) was within the normal range for sleep in healthy adults [17], our results show a large variation in sleep during night time, which may indicate either insomnia or hypersomnia after a stroke [14,18 ]. Only $52 \%$ of the patients had estimated sleep within what is recommended as normal sleep time per night (6-8 hours) in an adult population [19].

The high WASO and WAKES per night in this sample raises the question of whether sleep during the acute phase after a stroke can be considered restorative, when the normal WASO is less than $10 \%$ of TST and people normally are awaken fewer than six times a night [17]. In general, actigraphy has been criticized for overestimating TST and underestimating WAKES [20] especially in populations with fragmented sleep. In a comparison study between PSG and actigraphy, it is shown that WAKES are difficult to detect if 
the subject lies immobile with an actigraph [21]. Especially older people may lay still and awake at night time. If this is the case, in our study, the TST may be lower and the number of WAKES and the WASO may be even higher than our results have shown. However, our results are consistent with PSG findings $[1,3,6,18]$, in which stroke patients had higher WASO than age and gender-matched controls.

It has been demonstrated that memory and attention decrease as WASO increases [6], and so disrupted sleep may result in poor sleep quality. The sample in our study was predominantly older patients, and it can be argued that older individuals wake more often than the general population. However WAKES and WASO differed considerably from normal values. Patients who slept in the hospital had more WAKES than those who slept at home all three nights. The effect size of this relationship was medium, indicating a probable clinical significance [16]. Hospital environment is often known to have higher levels of noise, light, and interruptions that may disturb sleep. By objective measures, sleeping in hospital resulted in more fragmented, and thus less restorative sleep than sleeping at home in our sample, but there was no difference by self-report measure. If the medical and practical conditions at home are favorable, it may be better for the patient's sleep quality to sleep at home even in the acute phase after a stroke. Restorative sleep may promote the rehabilitation process in stroke patients.

Daytime estimated sleep was related to a longer hospital stay, but this relationship was not evident after controlling for sociodemographic, clinical, and contextual variables and self-reported sleep. However, daytime sleepiness is associated with nonrestorative sleep and deterioration in performance even in a general population [7]. More daytime sleep (by observation and wrist actigraphy) has been reported in a study of patients undergoing rehabilitation and is linked to less immediate functional recovery in these patients [22]. An overestimation of sleep during daytime may be suspected in immobile patients, as with TST. The mean sleep during daytime was 85 minutes and was not surprisingly high, but the variation was large. It remains to explore whether sleep during daytime is a sign of passivity or a way to compensate for disturbed sleep at night.

The self-reported sleep quality (PSQI) score averaged 6.9, which is above the cut point used in most populations ( 5 on a $0-21$ scale) to indicate poor sleep [12]. The patient's selfreported sleep quality correlated with the estimated TST and WASO from their actigraphy data.

Even though it is well known that men have poorer sleep quality as they age (less slow wave sleep) compared to women of similar age [23], this might not be considered in clinical practice. Men in our study had less sleep than women during night time, and this might be a consequence of age but remain an important finding. Sleep hygiene interventions, such as avoiding caffeine before bedtime, use of hypnotic agents, decreasing arousal and light at night should be gender-tailored strategies. Patient and family education about general sleep hygiene could be beneficial for improving the restorative function of sleep during the acute phase after a stroke [24].
4.1. Strengths and Limitations. Empirical sleep studies of stroke patients using more invasive polysomnography methods in laboratory settings have often focused on sleepdisordered breathing, have assessed only night time sleep in a sleep laboratory, or have examined only in small samples [6]. Because the actigraph is the weight and size of a wristwatch, it is assumed that this method of monitoring has little impact on a patient's natural activities and sleep patterns. Furthermore, an actigraph collects data that estimates sleep also during the day. The actigraph variables reported here are in accordance with the recommended standards [25] which should allow our findings to be compared with those of future studies. As mentioned in the discussion, we are aware of the weaknesses and limitations of actigraphs, but we agree that actigraphy can give important information in mapping the stroke patient sleep and wake pattern [5].

This study had a descriptive and correlational design and we were not able to compare our findings with a control group. Comparison between our stroke sample and an age matched control group would have strengthened the study design with regards to interpretation of the findings. Since only 6 of the patients had hemorrhagic stroke the results cannot be generalized to all stroke types and the result may be suffering a type II error.

We did not have data on how many of the patients were sharing their hospital room, or if the room-mates disturbed each patient's sleep. This may have been a confounder in estimating the sleep-wake pattern, especially of those who slept in hospital. Because the inclusion criteria included being able to speak and answer questions, patients with severe stroke were not represented in this study. This is further emphasized by the fact that $51 \%$ percent of the patients slept at home for one, two, or all three nights. Since the patients with the most severe disabilities probably are excluded from this sample, our findings cannot be generalized to those patients. Data on the prestroke sleep patterns or self-care levels of the participants were not available. Such data, if available, would allow the examination of changes in the sleeping experience in the poststroke period. One area of research suggested by the findings of this study is the impact of sleep quality on activities of daily living and dependence in the acute phase after a stroke.

\section{Conclusion}

The variability in our results indicates that both hypersomnia and insomnia may occur in the acute phase after first-ever stroke. Estimated sleep was especially disturbing in men regardless of age, and heath care workers should pay special attention to men "s" poor sleep-wake patterns during the acute phase while in hospital. The number of times the patients woke and the amount of waking time during nights were considerable. Further research is needed to assess a possible relationship between sleep-wake patterns in the acute phase and the patient's' participation in the rehabilitation process.

\section{Acknowledgments}

This paper is a product stemming from the research project: poststroke fatigue for which H.S. Kim is the project director 
and 6. Eilertsen, A. Lerdal, and H. Ormstad are the principal researchers. This project is funded by the Research Council of Norway for 2007 to 2010. The authors acknowledge the support and assistance provided by various staff members of Buskerud Hospital in Drammen and Aker Hospital in Oslo, Norway in carrying out this research project, especially Gunn Pedersen who has worked as a research assistant and participated with data collection. A. Lerdal has received funding from the RCN (Grant no. 19256) and the U.S.Norway Fulbright Foundation.

\section{References}

[1] A. Terzoudi, T. Vorvolakos, I. Heliopoulos, M. Livaditis, K. Vadikolias, and H. Piperidou, "Sleep architecture in stroke and relation to outcome," European Neurology, vol. 61, pp. 16-22, 2009.

[2] C. L. Bassetti and M. S. Aldrich, "Sleep electroencephalogram changes in acute hemispheric stroke," Sleep Medicine, vol. 2, no. 3, pp. 185-194, 2001.

[3] C. Müller, P. Achermann, M. Bischof, A. C. Nirkko, C. Roth, and C. L. Bassetti, "Visual and spectral analysis of sleep EEG in acute hemispheric stroke," European Neurology, vol. 48, no. 3, pp. 164-171, 2002.

[4] J. Vock, P. Achermann, M. Bischof et al., "Evolution of sleep and sleep EEG after hemispheric stroke," Journal of Sleep Research, vol. 11, no. 4, pp. 331-338, 2002.

[5] D. M. Hermann, M. Siccoli, P. Brugger et al., "Evolution of neurological, neuropsychological and sleep-wake disturbances after paramedian thalamic stroke," Stroke, vol. 39, no. 1, pp. 62-68, 2008.

[6] M. M. Siccoli, N. Rölli-Baumeler, P. Achermann, and C. L. Bassetti, "Correlation between sleep and cognitive functions after hemispheric ischaemic stroke," European Journal of Neurology, vol. 15, no. 6, pp. 565-572, 2008.

[7] M. M. Ohayon, "Prevalence and correlates of nonrestorative sleep complaints," Archives of Internal Medicine, vol. 165, no. 1, pp. 35-41, 2005.

[8] D. Foley, S. Ancoli-Israel, P. Britz, and J. Walsh, "Sleep disturbances and chronic disease in older adults: results of the 2003 National Sleep Foundation Sleep in America Survey," Journal of Psychosomatic Research, vol. 56, no. 5, pp. 497-502, 2004.

[9] K. A. Lee, C. Landis, E. R. Chasens et al., "Sleep and chronobiology: recommendations for nursing education," Nursing Outlook, vol. 52, no. 3, pp. 126-133, 2004.

[10] World Health Organisation, The ICD-10 Classification of Mental and Behavioural Disorders: Clinical Descriptions and Diagnostic Guidelines, WHO, Geneva, Switzerland, 1992.

[11] J. Ware, K. K. Snow, M. Kosinski, and B. Gandek, SF-36 Health Survey: Manual \& Interpretation Guide, The Health Institute, New England Medical Center, Boston, Mass, USA, 1997.

[12] D. J. Buysse, C. F. Reynolds III, T. H. Monk, S. R. Berman, and D. J. Kupfer, “The Pittsburgh Sleep Quality Index: a new instrument for psychiatric practice and research," Psychiatry Research, vol. 28, no. 2, pp. 193-213, 1989.

[13] S. L. Beck, A. L. Schwartz, G. Towsley, W. Dudley, and A. Barsevick, "Psychometric evaluation of the Pittsburgh Sleep Quality Index in cancer patients," Journal of Pain and Symptom Management, vol. 27, no. 2, pp. 140-148, 2004.

[14] S. Pallesen, I. H. Nordhus, S. Omvik, B. Sivertsen, and B. Bjorvatn, "Pittsburgh Sleep Quality Index," Tidsskrift for Norsk Psykologforening, pp. 714-717, 2005.
[15] S. Ancoli-Israel, R. Cole, C. Alessi, M. Chambers, W. Moorcroft, and C. P. Pollak, "The role of actigraphy in the study of sleep and circadian rhythms," Sleep, vol. 26, no. 3, pp. 342-392, 2003.

[16] J. Cohen, “A power primer," Psychological Bulletin, vol. 112, no. 1, pp. 155-159, 1992.

[17] A. M. Berger, K. P. Parker, S. Young-McCaughan et al., "Sleep wake disturbances in people with cancer and their caregivers: state of the science," Oncology Nursing Forum, vol. 32, no. 6, pp. E98-E126, 2005.

[18] J. M. Gottselig, C. L. Bassetti, and P. Achermann, "Power and coherence of sleep spindle frequency activity following hemispheric stroke," Brain, vol. 125, no. 2, pp. 373-383, 2002.

[19] F. P. Cappuccio, L. D’Elia, P. Strazzullo, and M. A. Miller, "Sleep duration and all-cause mortality: a systematic review and meta-analysis of prospective studies," Sleep, vol. 33, no. 5, pp. 585-592, 2010.

[20] L. de Souza, A. A. Benedito-Silva, M. L. Pires, D. Poyares, S. Tufik, and H. M. Calil, "Further validation of actigraphy for sleep studies," Sleep, vol. 26, no. 1, pp. 81-85, 2003.

[21] C. Acebo and M. K. LeBourgeois, "Actigraphy," Respiratory Care Clinics of North America, vol. 12, no. 1, pp. 23-30, 2006.

[22] C. A. Alessi, J. L. Martin, A. P. Webber et al., "More daytime sleeping predicts less functional recovery among older people undergoing inpatient post-acute rehabilitation," Sleep, vol. 31, no. 9, pp. 1291-1300, 2008.

[23] D. L. Bliwise, "Normal aging," in Principles and Practice of Sleep Medicine, M. H. Kryger, T. Roth, and W. C. Dement, Eds., pp. 24-38, Elsevier Saunders, Philadelphia, Pa, USA, 4th edition, 2005.

[24] C. M. Morin, "Psychological and behavioral treatments for primary insomnia," in Principles and Practice of Sleep Medicine, pp. 726-731, 4th edition, 2005.

[25] A. M. Berger, K. K. Wielgus, S. Young-McCaughan, P. Fischer, L. Farr, and K. A. Lee, "Methodological challenges when using actigraphy in research," Journal of Pain and Symptom Management, vol. 36, no. 2, pp. 191-199, 2008. 


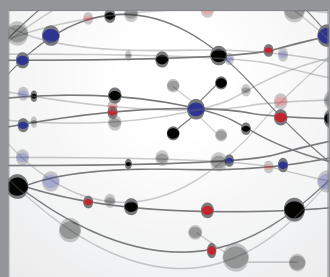

The Scientific World Journal
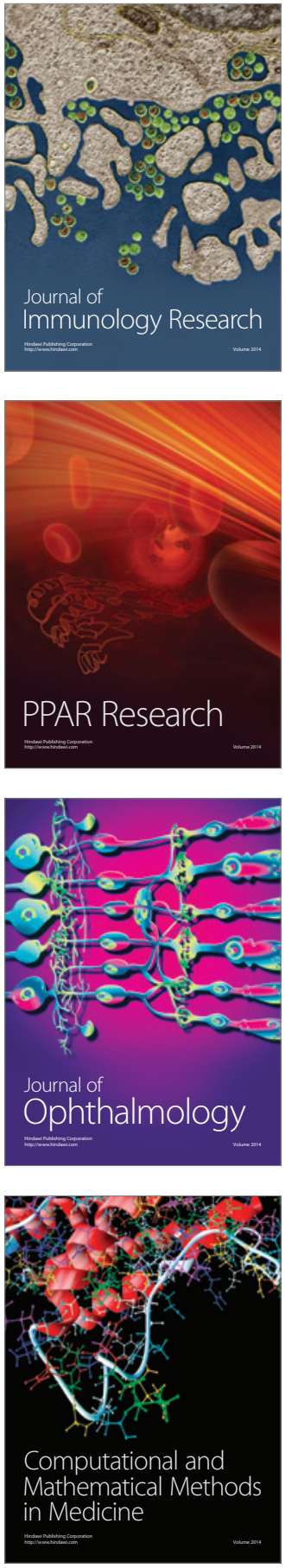

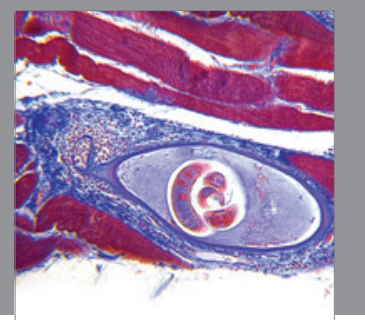

Gastroenterology

Research and Practice
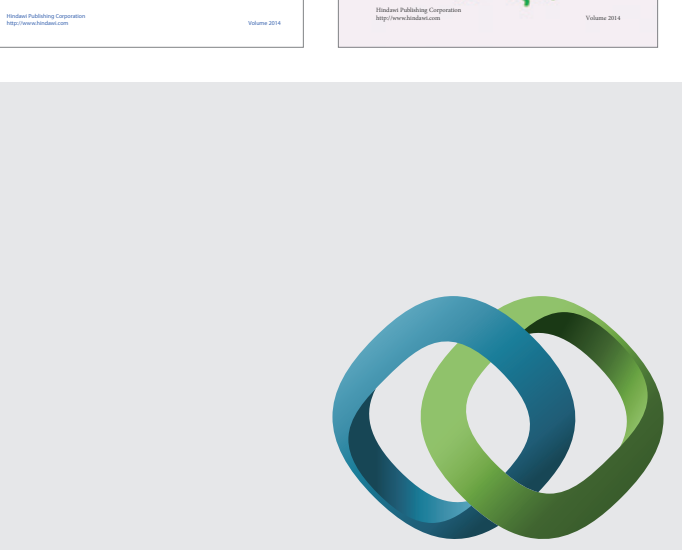

\section{Hindawi}

Submit your manuscripts at

http://www.hindawi.com
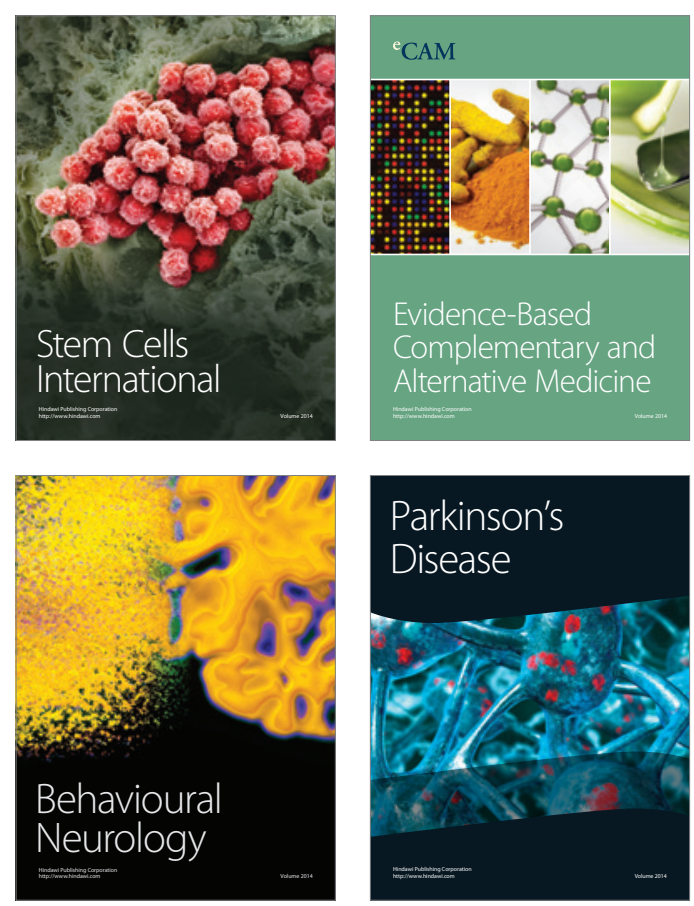

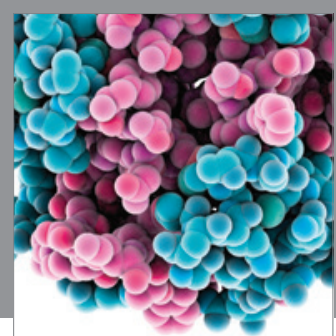

Journal of
Diabetes Research

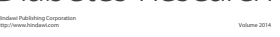

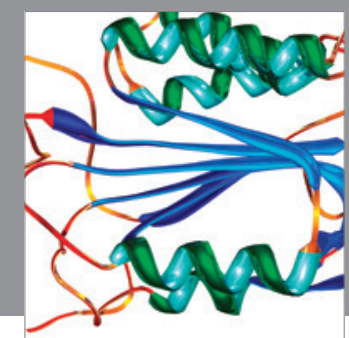

Disease Markers
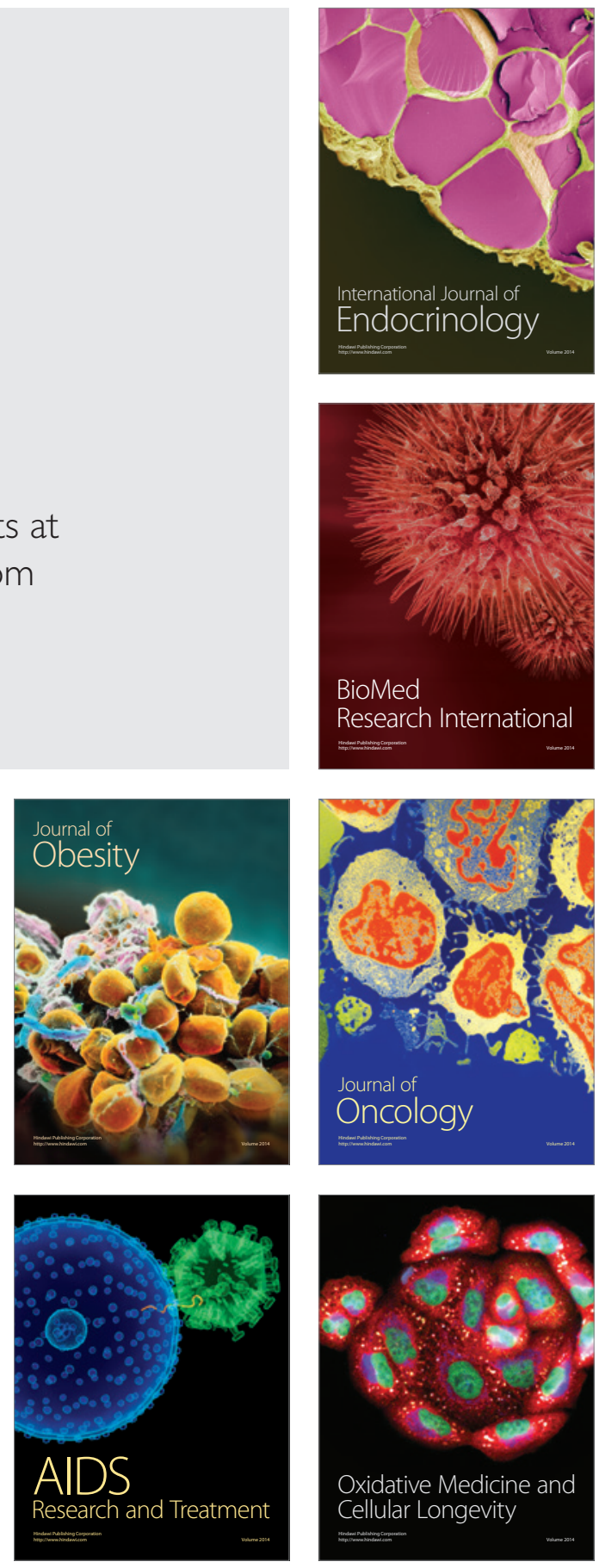